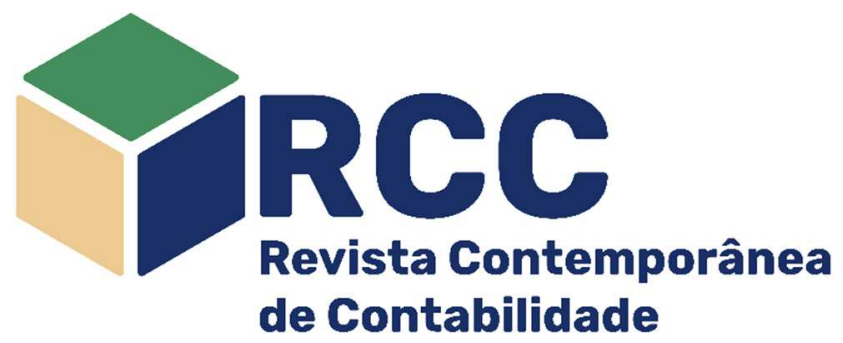

\title{
Fatores explicativos da gestão fiscal em municípios brasileiros
}

\author{
Explicative factors of the fiscal management in Brazilian municipalities
}

\section{Factores explicativos de la gestión fiscal en municipios brasileños}

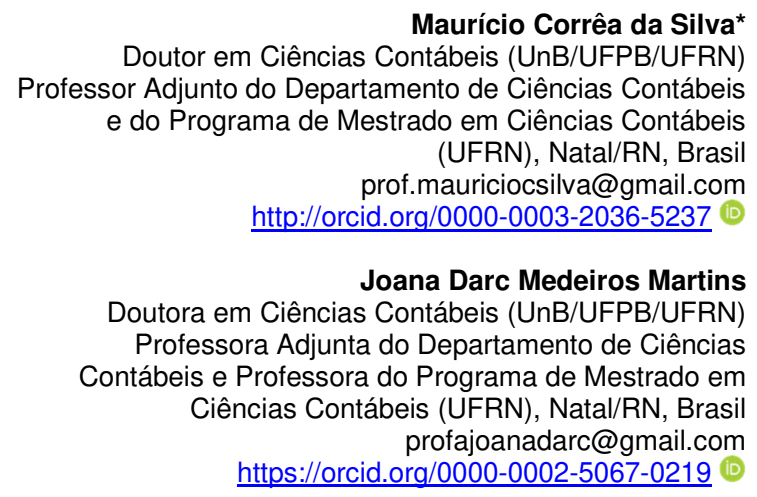

Maurício Corrêa da Silva* Doutor em Ciências Contábeis (UnB/UFPB/UFRN) Adjunto do Departamento de Ciências Contábeis (UFRN), Natal/RN, Brasil a@gmail.com

eiros Martins outora em Ciências Contábeis (UnB/UFPB/UFRN) e Professora do Programa de Mestrado em (UFR), Natal/RN, Brasil https://orcid.org/0000-0002-5067-0219 (i)
Fábia Jaiany Viana de Souza Doutora em Ciências Contábeis pela Universidade Federal da Paraíba (UFPB) Servidora do Instituto Federal de Educação, Ciência e Tecnologia do Rio Grande do Norte (IFRN), Nova Cruz/RN, Brasil fabiajaiany@yahoo.com.br https://orcid.org/0000-0001-5528-3229

Renata Paes de Barros Câmara Doutora em Engenharia Mecânica pela Escola de Engenharia Mecânica de São Carlos Professora Associada e do Doutorado e Mestrado em Ciências Contábeis (UFPB), João Pessoa/PB, Brasil rpbcamara@gmail.com https://orcid.org/0000-0001-6953-9811 (1)

Endereço do contato principal para correspondência* Av. José Rodrigues de Aquino Filho, 640 - Alto de Santa Luzia - CEP: 59215-000 - Nova Cruz, RN - Brasil

\begin{abstract}
Resumo
O objetivo desta investigação é analisar, dentre um conjunto de fatores, quais são os explicativos para a gestão fiscal evidenciada pelo Índice da Federação das Indústrias do Estado do Rio de Janeiro (FIRJAN) de Gestão Fiscal (IFGF) em municípios brasileiros. O estudo utiliza a estratégia de investigação da pesquisa avaliativa de resultados com o método empírico-analítico (banco de dados). Os resultados evidenciaram no modelo regressivo utilizado que as variáveis: Produto Interno Bruto - PIB (2013) per capita, Índice de Governança Municipal - IGM (2016) e a localização dos municípios nas regiões brasileiras foram significativas para explicar a gestão fiscal em municípios brasileiros. As variáveis número de habitantes (população) e o Índice FIRJAN de Desenvolvimento Municipal IFDM - (Edição 2015 - ano 2013) não foram significativas no modelo. O estudo contribui para a sociedade (os cidadãos) tomar ciência de fatores relacionados com gestão fiscal, além de contribuir para a literatura com uma forma diferenciada de análises com métrica quantitativa (modelo regressivo generalizado) e assim motivar mais estudos correlatos.
\end{abstract}

Palavras-chave: Gestão Fiscal; Fatores explicativos; Índices; Indicadores

\section{Abstract}

The purpose of this investigation is to analyze, among a set of factors, which may explain the fiscal management highlighted by the Fiscal Management Index of the Federation of Industries of the State of Rio de Janeiro (FIRJAN / IFGF) in Brazilian municipalities. The study uses the results-based evaluation research strategy with the empirical-analytical method (database). The results showed, in the regressive model used, that the variables: per capita Gross Domestic Product - GDP (2013), Municipal Governance Index - IGM (2016) and the location of municipalities in the Brazilian regions were significant to explain fiscal management in Brazilian municipalities. The variables number of inhabitants (population) and the FIRJAN Index of Municipal Development (IFDM) - (Edition 2015 - year 2013) were not significant in the model. The study enables society (the citizens) to become aware of factors related to fiscal management, in addition to contributing to the literature with a differentiated form of analysis with quantitative metrics (generalized regressive model) and 
thus motivating more correlated studies.

Keywords: Fiscal Management; Explicative factors; Indices; Indicators

\section{Resumen}

El objetivo de esta investigación es analizar, de entre un conjunto de factores, cuáles son los explicativos para la gestión fiscal evidenciada por el Índice de la Federación de las Industrias del Estado de Rio de Janeiro (FIRJAN) de Gestión Fiscal (IFGF) en municipios brasileños. El estudio utiliza la estrategia de investigación de la pesquisa de evaluación de resultados con el método empírico-analítico (banco de datos). Los resultados evidenciaron en el modelo regresivo utilizado que las variables: Producto Interno Bruto - PIB (2013) per capita, Índice de Gobernanza Municipal - IGM (2016) y la localización de los municipios en las regiones brasileñas fueron significativas para explicar la gestión fiscal en municipios brasileños. Las variables número de habitantes (población) y el Índice FIRJAN de Desarrollo Municipal IFDM - (Edición 2015 - año 2013) no fueron significativas en el modelo. El estudio contribuye para la sociedad (los ciudadanos) tener información de factores relacionados con gestión fiscal, además de contribuir para la literatura con una forma diferenciada de análisis con métrica cuantitativa (modelo regresivo generalizado) y así motivar más estudios correlatos.

Palabras clave: Gestión Fiscal; Factores explicativos; Índices; Indicadores

\section{Introdução}

A Federação das Indústrias do Estado do Rio de Janeiro (FIRJAN) desenvolveu o Índice FIRJAN de Gestão Fiscal (IFGF) com o objetivo de estimular a cultura da responsabilidade administrativa, possibilitando aprimoramento da gestão fiscal dos municípios, bem como o aperfeiçoamento das decisões dos gestores públicos quanto à alocação dos recursos. O IFGF é composto por cinco indicadores: Receita Própria, Gastos com Pessoal, Investimentos, Liquidez e Custo da Dívida. Os quatro primeiros possuem peso 22,5\% e o último $10,0 \%$ no resultado final do Índice. A pontuação varia entre 0 e 1, sendo que, quanto mais próxima de 1 , melhor a situação fiscal do município no ano em observação (FIRJAN, 2018).

A FIRJAN (2018) considera como conceito A (Gestão de Excelência), os resultados superiores a 0,8 pontos; conceito B (Boa Gestão) entre 0,6 e 0,8; conceito C (Gestão em Dificuldade) de 0,4 a 0,6 e conceito D (Gestão Crítica) para valores inferiores a 0,4. A edição do IFGF de 2017, ano de referência 2016, segundo a FIRJAN (2018), avaliou a situação fiscal de 4.544 municípios, onde vivem 177,8 milhões de pessoas $87,5 \%$ da população brasileira. Apesar da determinação da lei, os dados do exercício fiscal 2016 de 1.024 prefeituras não estavam disponíveis ou não eram consistentes (informações que não foram passíveis de análise).

O IFGF nas pesquisas acadêmicas é abordado em diversos focos: gestão de pequenos municípios (KLERING; KRUEL; STRANZ, 2012); relações entre indicadores socioeconômicos e a gestão fiscal dos municípios maranhenses com uso da análise fatorial (PEREIRA et al., 2013). O IFGF foi utilizado como variável independente para verificar as características explicativas do nível de disclosure voluntário de municípios do Estado de Minas Gerais em seus portais eletrônicos (AVELINO et al., 2014); relações entre variáveis socioeconômicas e a gestão dos municípios do Estado de Minas Gerais em que os indicadores do IFGF foram utilizados como variáveis dependentes com uso de regressão linear múltipla (COSTA; TAVARES, 2014).

Melo, Souza e Bonfim (2015) utilizaram o IFGF como proxy de equilíbrio para verificar relações em que medida a baixa participação eleitoral, a competição política, bem como as alianças eleitorais influenciaram na variabilidade do equilíbrio fiscal da gestão municipal. Bernardo, Reis e Sediyama (2017) utilizaram variáveis socioeconômicas, demográficas e financeiras (incluindo o IFGF) para verificarem a existência de características explicativas do nível de transparência na administração pública em municípios de médio e grande porte no Estado de Minas Gerais.

Questiona-se assim: Quais são os fatores explicativos da gestão fiscal em municípios brasileiros evidenciada pelo Índice FIRJAN de Gestão Fiscal (IFGF)? Dessa forma, o objetivo desta investigação é analisar, dentre um conjunto de fatores, quais são os explicativos para a gestão fiscal, tendo como proxy o Índice da Federação das Indústrias do Estado do Rio de Janeiro (FIRJAN) de Gestão Fiscal (IFGF) em municípios brasileiros.

O estudo justifica-se pela relevância em que a gestão fiscal tem nos debates acadêmicos (estudos divulgados) e para os gestores públicos e a sociedade. A sociedade tem o direito de participar e fiscalizar a gestão pública. Contudo, existe uma assimetria informacional, que com base na Teoria da Agência (aplicada ao setor público), pode ser analisada: os cidadãos desconhecem a gestão pública e são os titulares (principal) e os gestores públicos (os agentes) que conhecem essa gestão. Dessa forma, as informações dos resultados de pesquisas avaliativas podem reduzir as assimetrias, e assim, pode-se ampliar a possibilidade do exercício do controle social nos governos locais com o efeito da ação dos cidadãos participantes sobre as atividades do Estado. Espera-se também, motivar outros pesquisadores a realizarem mais pesquisas de avaliação de resultados das entidades públicas.

Quanto à literatura, esta investigação aborda o tema como uma forma diferenciada das análises com métrica quantitativa das observadas nas pesquisas de Costa e Tavares (2014) que utilizaram o IFGF e seus 
indicadores como variáveis dependentes para associar com variáveis socioeconômicas; Avelino et al. (2014) - IFGF como variável independente para explicar características explicativas do nível de disclosure e Shikida e Faria e Araújo Jr. (2014) - IFGF como variável independente para explicar a criação de novos estados. Também difere do estudo de Cavalcante (2015) - IFGF e seus indicadores como variáveis dependentes para associar com variáveis eleitorais (competição; coalizões; partido do governador etc.) e Melo, Souza e Bonfim (2015) - IFGF como variável dependente para associar com variáveis eleitorais (abstenção; partidos; coligações etc.). Na pesquisa de Melo e Silva (2016), o IFGF é utilizado como variável independente para explicar capacidade administrativa na educação básica e Bernardo, Reis e Sediyama (2017) - IFGF como uma das variáveis independentes para explicar nível de transparência.

Para atingir o objetivo proposto, esta investigação está amparada pela Teoria da Agência aplicada ao setor público (assimetria da informação), além de referencial teórico sobre desempenho de entidades públicas. O artigo está dividido em cinco seções. Após esta introdução, a seção dois traz a revisão da literatura. A seção seguinte os procedimentos metodológicos. A quarta seção mostra os resultados da pesquisa e análises. A quinta seção trata das considerações finais.

\section{Revisão da Literatura}

\subsection{Teoria da Agência aplicada ao setor Público}

$\mathrm{Na}$ Teoria da Agência formalizada no artigo seminal de Jensen e Meckling (1976), a sociedade é concebida como uma rede de contratos, explícitos e implícitos, os quais estabelecem as funções e definem os direitos e deveres de todos os participantes. Neste contexto, aparecem as figuras do principal (centro de todos os interesses na empresa) e o agente (que é alguém contratado para gerir a empresa). Dentro dessa relação, entre principal e agente, ocorre uma assimetria de informações, pois o agente tem acesso a dados que o principal não tem e vice-versa. Assim, a hipótese fundamental dessa teoria é que as pessoas têm interesses diferentes e cada uma busca maximizar seus próprios objetivos.

Para Dawson et al. (2016), a Teoria da Agência pode ser aplicada ao setor público, embora seja necessário um entendimento-chave dos atores envolvidos. Ao entender que a governança não precisa ser um jogo de soma zero em que o principal ganha e o agente perde ou vice-versa, uma mudança intelectual pode ser feita para maximizar os resultados positivos em vez de simplesmente tentar evitar resultados negativos. Dado o tamanho e a amplitude dos serviços oferecidos pelo Estado e a crítica dos serviços pelos cidadãos, tal mudança poderia ter impactos profundos sobre a qualidade das vidas daqueles a quem os governos servem.

Segundo Malmir et al. (2014), a Teoria da Agência pode ter o efeito de criar uma nova administração pública por destacar questões em relação às responsabilidades das estruturas estatais que distingue das estruturas privadas. Esta teoria faz com que os agentes atuem e sejam responsáveis perante os interesses de seus cidadãos. Ao aplicar os princípios da estrutura da teoria, o comportamento de indivíduos em diferentes níveis hierárquicos da organização, representam variáveis importantes que afetam esses mecanismos de monitoramento de custos, incentivos e controle.

Suzart (2012) afirma que os cidadãos delegam poderes aos gestores públicos para que estes realizem ações governamentais que atendam os interesses sociais e promovam o bem-estar à população, surgindo assim entre a sociedade e os governantes uma relação de agência. Para o autor, embasado na Teoria da Agência, pode-se afirmar que há evidências de que os gestores públicos, ao invés de atender aos anseios sociais, podem buscar saciar interesses pessoais. Isto pode ser percebido no aumento no número de notícias sobre desvios e má gestão de recursos públicos, nas últimas duas décadas, tanto no cenário brasileiro, quanto no internacional.

O problema da agência decorre dos interesses divergentes entre o principal e o agente e da existência da assimetria da informação, em razão do problema de surgir no momento em que as informações disponíveis são desiguais entre os participantes. O esforço do agente é um componente necessário da função de produção, mas pode implicar em alguma desutilidade. Ele pode tirar vantagem injusta de suas informações superiores se as condições externas forem favoráveis (LERUTH; PAUL, 2006; ARROW, 1984).

Rocha et al. (2012) argumentam que a contabilidade pode auxiliar a reduzir a assimetria da informação, no momento em que são divulgadas as demonstrações financeiras atualizadas da organização, observado que os investidores não possuem o mesmo nível de acesso às informações que os administradores da entidade. Para os autores, os teóricos trabalhavam com o modelo de firma da teoria clássica, até que a Teoria da Agência rompeu com esse paradigma. Na teoria clássica não existe conflito de interesse e na Teoria da Agência existe.

Assim, diante da importância dos cidadãos em obter informações acerca da gestão pública que é financiada com seus recursos, torna-se fundamental a realização de pesquisas avaliativas com o intuito de esclarecer resultados de ações governamentais, bem como de identificar fatores relacionados com essa gestão. A Teoria da Agência aplicada ao setor público permite analisar os interesses do agente (gestores públicos) e da sociedade (principal), além de motivar que haja esclarecimentos sobre a gestão pública para diminuir a assimetria informacional. 


\subsection{Avaliação de desempenho de entidades públicas}

A avaliação de desempenho consiste num exame regular e cuidadoso da implementação das políticas públicas e análise dos resultados alcançados (JULNES, 2006). As dificuldades relativas à mensuração de desempenho no setor público são substantivas, tanto no Brasil como nas experiências internacionais. Não se trata de atitudes intencionais voltadas à manipulação de dados ou ao falseamento dos resultados; são, antes, dificuldades ligadas à natureza das atividades desempenhadas pelo Estado (PACHECO, 2009).

A utilização de indicadores de desempenho para aferir os resultados alcançados pelos administradores é uma metodologia que está relacionada ao conceito de gerenciamento voltado para resultados. Na auditoria operacional, os indicadores de desempenho são usados para medir economicidade, eficácia, eficiência e efetividade, conforme esclarecimentos do Tribunal de Contas da União - TCU (BRASIL, 2010).

Figueiredo Filho et al. (2013) esclarecem que os indicadores permitem estimar a efetividade das ações governamentais e avaliar em que medida o dinheiro público está sendo eficientemente utilizado. A construção e a divulgação de indicadores sociais têm um papel fundamental no planejamento e execução das ações públicas. Para Tshukudu (2014), o gerenciamento de desempenho não ocorre no vácuo. É essencial que uma cultura organizacional seja analisada para ver em quais aspectos promove ou dificulta 0 desempenho efetivo. A cultura organizacional deve ser criada e reforçada para criar um ambiente de alto desempenho.

Mecanismos de accountability, de acordo com Schillemans (2015), estão entre os meios mais importantes com que os governos protegem e melhoram o desempenho das organizações do setor público e são de importância crucial nas democracias. A prestação de contas é uma contingência generalizada na vida profissional de gestores públicos e dos profissionais do setor público.

Segundo Caiden e Caiden (2001), Costa e Castanhar (2003) e Secchi (2013), para avaliar o desempenho dos programas e os resultados das ações do governo é necessário estabelecer os critérios de avaliação. Costa e Castanhar (2003) esclarecem que os principais critérios são: eficiência; eficácia; impacto ou efetividade; sustentabilidade; análise custo-efetividade; satisfação do beneficiário; equidade etc.

Secchi (2013) esclarece que os critérios de avaliação são operacionalizados por meio de indicadores e que estes são artifícios (proxies) que podem ser criados para medir inputs (recursos financeiros, humanos e materiais), outputs (produtividade de serviços/produtos) e outcomes (resultados). Os indicadores de inputs medem esforços e os indicadores de outputs e outcomes medem realizações.

$\mathrm{Na}$ literatura são encontrados diversos índices e indicadores utilizados nas avaliações de resultados da gestão pública, tais como: o Índice de Desenvolvimento Humano Municipal (IDHM) do Programa das Nações Unidas para o Desenvolvimento (PNUD, 2018), o Índice FIRJAN de Desenvolvimento Municipal (IFDM) da Federação das Indústrias do Estado do Rio de Janeiro (FIRJAN, 2018) e o Índice FIRJAN de Gestão Fiscal (IFGF) da Federação das Indústrias do Estado do Rio de Janeiro (FIRJAN, 2018). São exemplos também: o Índice de Oportunidades da Educação Brasileira (IOEB), organizado pelo Centro de Liderança Pública (CLP, 2018), o Ranking de Eficiência dos Municípios (REM- F), elaborado pela Folha de São Paulo (2018) e o Índice de Desenvolvimento da Educação Básica (IDEB), criado pelo Instituto Nacional de Estudos e Pesquisas Educacionais Anísio Teixeira (INEP, 2018).

Contudo, observa-se que existem críticas quanto ao processo de construção de índices. Na visão de Guimarães e Jannuzzi (2005), por mais rigorosas e criteriosas que aparentem ser as metodologias e práticas estatísticas utilizadas, suas hipóteses são muito arbitrárias e a operação de sintetização de indicadores sociais em um único índice é raramente apoiada em alguma teoria ou marco metodológico consistente.

Para Silva, Silva e Silva (2016), infelizmente ou felizmente (inviabilidade) não é possível realizar avaliações de todo o universo que envolve os resultados das gestões públicas. As avaliações são realizadas como aproximações (proxies) do universo, como por exemplo: a política educacional do ensino fundamental público é avaliada pelo Índice de Desenvolvimento da Educação Básica (IDEB), que é um indicador sintético (avalia os dados sobre aprovação escolar e das médias de desempenho nas avaliações).

Embora os indicadores/índices sejam considerados sintéticos (GUIMARÃES; JANNUZZI, 2005; SILVA; SILVA; SILVA, 2016), observa-se suas utilidades para compreender fatores (nível de disclosure, transparência etc.) relacionados com a gestão pública (KLERING; KRUEL; STRANZ, 2012; PEREIRA et al., 2013; AVELINO et al., 2014; COSTA; TAVARES, 2014). É muito dispendioso avaliar todo o universo das variáveis envolvidas no processo de avaliação de desempenho. Neste sentido, é que foram criados os indicadores/índices sintéticos.

Depreende-se que o IFGF pode ser considerado como um indicador sintético, haja vista que considera como gestão fiscal somente cinco áreas (indicadores): receita própria (receita própria dividida pela receita corrente líquida); gastos com pessoal (1 menos a razão entre os gastos com pessoal e a receita corrente líquida); investimentos (investimentos divididos pela receita corrente líquida); liquidez (1 menos a razão das despesas de restos a pagar e o ativo financeiro) e o custo da dívida (juros e amortizações divididos pela receita líquida real). A gestão fiscal envolve também ações planejadas e transparentes, metas, equilíbrios das contas públicas, limites de gastos, renúncias de receitas, dentre outras. 


\section{Procedimentos Metodológicos}

Esta investigação utiliza a estratégia da pesquisa de avaliação de resultados. Para Martins e Theóphilo (2009), essa é uma estratégia empírica aplicada para avaliar programas, projetos, políticas etc. O método de pesquisa utilizado é o empírico-analítico (arquivo/empirista - banco de dados) que corresponde à utilização de técnica de coleta, tratamento e análise de dados com métricas quantitativas. Matias-Pereira (2012) esclarece que nesse método, o processo de validação da prova científica é feito por meio de testes dos instrumentos, graus de significância e sistematização das definições operacionais.

A Teoria da Agência constitui o elemento teórico para suportar as análises de fatores explicativos da gestão fiscal em municípios brasileiros que podem ser utilizados para minimizar a assimetria informacional. Cabe ressaltar que não foram utilizadas variáveis e hipóteses específicas para verificar as associações com a teoria. Presume-se pela relação entre principal-agente (foco essencial da teoria) que quanto mais informações os cidadãos possuírem da gestão pública, mais condições terão de exercer um dos seus direitos: o controle social. O estudo de Suzart (2012) utilizou a Teoria da Agência sem testar nenhuma variável específica para analisar a atuação das instituições superiores de auditoria.

A população, desta investigação, abrange todos os 5.570 (cinco mil, quinhentos e setenta) municípios brasileiros, conforme o Instituto Brasileiro de Geografia e Estatística (IBGE) do ano de 2016 (IBGE, 2017). Para compor a amostra foram selecionados os 82 municípios brasileiros (Quadro 1) posicionados por ordem do número de habitantes (população) no ano de 2016 e que possuía todos os dados necessários da pesquisa. Para minimizar a diferença de tamanhos dos municípios da amostra nos resultados da investigação, com exceção da variável população, foram utilizadas variáveis de índices (IFDM e IGM) e dados per capita (PIB).

\section{Quadro 1 - Municípios da amostra da pesquisa}

\begin{tabular}{|c|c|c|}
\hline $\begin{array}{l}\text { São Paulo (SP); Rio de Janeiro } \\
\text { (RJ); Salvador (BA); Fortaleza (CE); } \\
\text { Belo Horizonte (MG); Manaus (AM); } \\
\text { Curitiba (PR); Recife (PE); Porto } \\
\text { Alegre (RS); Goiânia (GO); Belém } \\
\text { (PA); Guarulhos (SP); Campinas } \\
\text { (SP); São Luís (MA); Maceió (AL); } \\
\text { Duque de Caxias (RJ); Natal (RN); } \\
\text { Campo Grande (MS); Teresina (PI); } \\
\text { São Bernardo do Campo (SP); João } \\
\text { Pessoa (PB); Osasco (SP); São } \\
\text { José dos Campos (SP); Jaboatão } \\
\text { dos Guararapes (PE); Uberlândia } \\
\text { (MG); Contagem (MG); Sorocaba } \\
\text { (SP); }\end{array}$ & $\begin{array}{l}\text { Aracaju (SE); Feira de Santana } \\
\text { (BA); Cuiabá (MT); Joinville (SC); } \\
\text { Juiz de Fora (MG); Londrina (PR); } \\
\text { Aparecida de Goiânia (GO); Porto } \\
\text { Velho (RO); Ananindeua (PA); } \\
\text { Niterói (RJ); Belford Roxo (RJ); } \\
\text { Serra (ES); Vila Velha (ES); Caxias } \\
\text { do Sul (RS); Macapá (AM); Mauá } \\
\text { (SP); São José do Rio Preto (SP); } \\
\text { Santos (SP); Mogi das Cruzes (SP); } \\
\text { Betim (MG); Campina Grande (PB); } \\
\text { Jundiaí (SP); Maringá (PR); } \\
\text { Piracicaba (SP); Olinda (PE); } \\
\text { Anápolis (GO); Bauru (SP); Vitória } \\
\text { (ES); }\end{array}$ & $\begin{array}{l}\text { Caucaia (CE); Vitória da Conquista } \\
\text { (BA); Blumenau (SC); Pelotas (RS); } \\
\text { Canoas (RS); Ponta Grossa (PR); } \\
\text { Petrolina (PE); Boa Vista (RR); } \\
\text { Paulista (PE); Uberaba (MG); } \\
\text { Cascavel (PR); Guarujá (SP); } \\
\text { Taubaté (SP); Praia Grande (SP); } \\
\text { Limeira (SP); Petrópolis } \\
\text { (RJ);Santarém (PA); Camaçari } \\
\text { (BA); Mossoró (RN); Suzano (SP); } \\
\text { Palmas (TO); Governador } \\
\text { Valadares (MG); Santa Maria (RS); } \\
\text { Taboão da Serra (SP); Gravataí } \\
\text { (RS); Várzea Grande (MT) e } \\
\text { Sumaré (SP). }\end{array}$ \\
\hline
\end{tabular}

Fonte: adaptado do IBGE (2017).

As variáveis (fatores) escolhidas para analisar a associação (relação) com o IFGF do ano de 2016, constam no Quadro 2. O ano de 2016 foi escolhido por ser o último em que consta todas as informações requeridas. O Produto Interno Bruto per capita de 2013 e o IFDM (Edição 2015) de 2013 foram utilizados como proxies pela falta de dados do ano de 2016. A delimitação do aspecto temporal de um ano é suficiente para realizar as análises (modelo de regressão utilizado). Convém, ainda, relatar que os dados da pesquisa foram coletados no mês de janeiro de 2018. A coleta de dados foi realizada dos sítios eletrônicos da Federação de Indústrias do Estado do Rio de Janeiro (FIRJAN, 2018), do Instituto Brasileiro de Geografia e Estatística (IBGE, 2017) e do Conselho Federal de Administração (CFA, 2018).

A variável dependente IFGF não apresenta endogenia com as variáveis explicativas. É composta pelos indicadores: receita própria, gastos com pessoal, investimentos, liquidez e custo da dívida. A variável independente IFDM acompanha anualmente o desenvolvimento socioeconômico em três áreas de atuação: emprego e renda, educação e saúde. A escolha dos fatores/variáveis independentes (Quadro 2) está fundamentada na literatura que analisam determinantes da transparência (CRUZ, 2010; TEJEDO-ROMERO; ARAÚJO (2018), e do desempenho da gestão pública (MENDES, SOUSA, 2006; GEMMELL; KNELLER; SANZ, 2016; CFA, 2018) como proxy para explicar fatores associados com a gestão fiscal dos municípios.

Mendes e Sousa (2006) estimaram a demanda por gastos públicos para os municípios brasileiros. Os resultados mostraram que, os municípios pertencentes às regiões Nordeste e Sudeste, tendem a apresentar maiores níveis de despesa por razões distintas. No caso do Nordeste, os serviços públicos são geralmente os únicos disponíveis para a população com menor poder aquisitivo, como consequência ocorre a elevação dos gastos. Na região Sudeste, a característica mais urbana contribui para o aumento da demanda por mais serviços públicos.

Para Cruz (2010), as variáveis: localização, participação em redes de integração municipal, PIB per capita, receita orçamentária, IDH-M, taxa de alfabetização, Índice FIRJAN de Desenvolvimento Municipal (IFDM) e o dinamismo municipal exercem influência direta e significativa sobre o nível de transparência da gestão pública municipal. Gemmell, Kneller e Sanz (2016) examinaram os efeitos das despesas públicas no 
PIB para o longo prazo, concentrando-se na despesa total e nas categorias específicas de gastos, nos países que compõem a OCDE (Organização para a Cooperação e Desenvolvimento Econômico). O estudo considerou as possíveis relações endógenas que possam existir entre despesa e crescimento econômico. Os resultados indicaram efeitos positivos nos níveis do Produto Interno Bruto relacionados à função educação, transporte e comunicação.

Quadro 2 - Descrição dos fatores/variáveis para análise de associação (relação)

\begin{tabular}{|c|c|c|c|}
\hline \multicolumn{2}{|c|}{ Fatores/variáveis } & Fonte & Expectativa \\
\hline \multicolumn{4}{|c|}{ Variável dependente (Y) - explicada } \\
\hline \multicolumn{2}{|c|}{ IFGF (2016) } & \multicolumn{2}{|l|}{ FIRJAN } \\
\hline \multicolumn{4}{|c|}{ Variáveis independentes $(X)$ - explicativas } \\
\hline \multicolumn{2}{|c|}{ Quantidade de Habitantes (População = POP) } & IBGE & Positiva \\
\hline \multicolumn{2}{|c|}{ PIB (2013) per capita } & IBGE & Positiva \\
\hline \multicolumn{2}{|c|}{ IFDM (Edição 2015 - ano 2013) } & FIRJAN & Positiva \\
\hline \multicolumn{2}{|l|}{ IGM (2016) } & CFA & Positiva \\
\hline \multirow{5}{*}{$\begin{array}{l}\text { Localização: } \\
\text { Regiões Brasileiras }\end{array}$} & Norte & \multirow{5}{*}{ IBGE } & \multirow{5}{*}{$\begin{array}{l}\text { Positiva pela ordem: Sudeste, Sul, } \\
\text { Nordeste, Centro-Oeste e Norte }\end{array}$} \\
\hline & Nordeste & & \\
\hline & Sudeste & & \\
\hline & Sul & & \\
\hline & Centro-Oeste & & \\
\hline
\end{tabular}

Legenda: FIRJAN = Federação das Indústrias do Estado do Rio de Janeiro; IFGF = Índice FIRJAN de Gestão Fiscal; PIB = Produto Interno Bruto; IFDM = Índice FIRJAN de Desenvolvimento Municipal; IBGE = Instituto Brasileiro de Geografia e Estatística; IGM = Índice de Governança Municipal; CFA = Conselho Federal de Administração;

Fonte: dados da pesquisa.

De acordo com Tejedo-Romero e Araújo (2018), as pressões exercidas pelos cidadãos e outras partes interessadas estão levando municípios a tornarem-se mais transparentes no processo de criação de legitimidade antes cidadãos e partes interessadas. Os municípios, onde os cidadãos têm maior acesso à Internet, têm mais população, a pressão fiscal é mais pesada e é governada pela esquerda, as partes interessadas são mais pressionadas para serem transparentes.

O Índice de Governança Municipal - IGM (CFA, 2018) consiste em uma métrica da governança pública nos municípios brasileiros a partir de três dimensões: finanças, gestão e desempenho. O índice foi elaborado a partir de dados secundários, e considera áreas como saúde, educação, gestão fiscal, habitação, recursos humanos, transparência, violência dentre outras. Para os gestores municipais, o índice pode ajudar a visualizar as necessidades e/ou boas práticas de sua região e para os cidadãos estimula-se a participação social, contribuindo ainda para maior integração do governo com a sociedade.

A utilização de apenas um ano das séries históricas existentes das variáveis é significativa para o estudo, haja vista que a técnica estatística utilizada (Modelo Linear Generalizado) assim o requer. Os resultados da pesquisa são restritos para a amostra. Não pode generalizar para os demais municípios (limitação do estudo).

As expectativas da investigação, com base nas variáveis escolhidas no Quadro 2, é que a quantidade de habitantes (POP), o PIB (2013) per capita, o IFDM (Edição 2015 - ano 2013), o IGM (2016) e a localização dos municípios nas regiões brasileiras (variáveis dummies - 1 pertence a região e 0 não) possam exercer positivamente uma associação (relação) para explicar os escores obtidos no IFGF. Estatisticamente, as hipóteses da pesquisa, são:

- Hipótese nula $\left(\mathbf{H}_{0}\right)$ : não existe associação (relação) entre as variáveis classificadas como explicativas no Quadro 1 com o IFGF;

- Hipótese alternativa $\left(\mathbf{H}_{1}\right)$ : existe associação (relação) entre as variáveis classificadas como explicativas no Quadro 1 com o IFGF.

A técnica utilizada na análise e interpretação dos dados escolhida foi a regressão do Modelo Linear Generalizado (MLG ou GLM em inglês: Generalized Linear Models). Os GLM foram consolidados do ponto de vista teórico e conceitual, por meio do trabalho seminal de Nelder e Wedderburn no ano de 1972, ao agruparem um grupo de modelos de regressão lineares e exponenciais não lineares, em que a variável dependente possui, por exemplo, distribuição normal, Bernoulli, binomial, Poisson ou Poisson-Gama (FÁVERO, 2015).

Um GLM é definido da seguinte forma:

$$
\eta_{1}=\alpha+\beta_{1} \cdot X_{1 i}+\beta_{2} \cdot X_{2 i} \quad \ldots+\beta_{k}, X_{k i}
$$

em que $\eta$ é conhecido por função de ligação canônica, $\alpha$ representa a constante, $\beta_{j}(j=1,2, \ldots ., k)$ são os coeficientes de cada variável explicativa e correspondem aos parâmetros a serem estimados, $X_{j}$ são as variáveis explicativas (métricas ou dummies) e os subscritos i representam cada uma das observações da amostra em análise $(i=1,2, \ldots, n$, em que $n$ é o tamanho da amostra). 
Existem três componentes para qualquer GLM (AGRESTI, 2007; MCCULLAGH, NELDER, 1989; OLSSON, 2002; CORDEIRO; DEMETRIO, 2008; MYERS et al., 2010):

- Componente aleatório - refere-se à distribuição de probabilidade da variável de resposta (Y); por exemplo, distribuição normal para $Y$ na regressão linear, ou distribuição binomial para $Y$ na regressão logística binária. Também chamado de modelo de ruído ou modelo de erro;

- Componente sistemático - especifica as variáveis explicativas $\left(X_{1}, X_{2}, \ldots X_{k}\right)$ no modelo, mais especificamente sua combinação linear na criação do chamado preditor linear; por exemplo, $\beta_{0}+\beta_{1} X_{1}+$ $\beta_{2} x_{2}$ como visto em uma regressão linear, ou como pode ser observado em uma regressão logística; $e$

- Função de ligação - $\eta$ ou $g(\mu)$ - especifica a ligação entre componentes aleatórios e sistemáticos. Ele diz como o valor esperado da resposta se relaciona com o preditor linear de variáveis explicativas; por exemplo, $\eta=g\left(E\left(Y_{i}\right)\right)=E\left(Y_{i}\right)$ para a regressão linear, ou $\eta=$ logit ( $\left.\pi\right)$ para a regressão logística.

Não existem restrições (pressupostos) para a utilização do GLM, exceto quanto ao tipo de distribuição utilizada (MCCULLAGH; NELDER, 1989; TURKMAN; SILVA, 2000; OLSSON, 2002; SWAN, 2006; AGRESTI, 2007; CORDEIRO; DEMÉTRIO, 2008; MYERS et al., 2010). Contudo, analisar a ausência da multicolinearidade das variáveis explicativas é importante para qualquer estimação quando se usa modelos regressivos. Os testes de significância, segundo Fávero (2015), são verificados com a qualidade de ajuste do modelo estimado (Goodness of Fit) com a desviância; o Teste de Omnibus (Qui-quadrado da razão de verossimilhança), parecido com o Teste $F$ na regressão linear múltipla e o Teste de Hipótese de Wald para analisar os $p$-valores (nível de significância) dos coeficientes $\beta$.

\section{Resultados e Discussões}

GLM.

A Tabela 1 apresenta os resultados das informações necessárias para utilizar a regressão no modelo

Tabela 1 - Informações do Modelo Linear Generalizado (GLM)

Variável dependente

Distribuição de probabilidade

Função de ligação

\begin{tabular}{lccc}
\hline Qualidade de ajustamento (Goodness of Fit) & Valor & $\mathrm{df}$ & $P$-valor \\
Desviância dimensionada & 82,461 & 72 & $0,1873421^{*}$ \\
\hline Teste de Omnibus & & $\mathrm{df}$ & $\mathrm{Sig}$. \\
Qui-quadrado da razão de verossimilhança & 39,229 & 9 & $0,000^{*}$ \\
\hline
\end{tabular}

Legenda: $\mathrm{df}$ = graus de liberdade; Sig. = nível de significância; * $=$ significância ao nível de $5 \%$.

Fonte: dados da pesquisa.

Observa-se na Tabela 1, que foi escolhida a distribuição de Tweedie e a função de ligação identidade para analisar os dados. Para Swan (2006), essa distribuição pertence à família exponencial de distribuições e fazem parte de um grupo maior de modelos chamado modelo linear generalizado. A família de distribuições de Tweedie é uma classe de distribuição que é capaz de modelar probabilidades discretas e contínuas juntas em um modelo.

A qualidade do ajuste do modelo estimado (Goodness of Fit) é adequada para $X^{2}$ cal (qui-quadrado calculado), haja vista que a desviância (deviance) apresentou valor maior que 0,05 ( $p$-valor de 0,187 - Tabela 1). Isto indica que não existem diferenças estatisticamente significantes entre os valores previstos e observados.

O qui-quadrado da razão de verossimilhança (Teste de Omnibus) obteve o nível de significância menor que 0,05 (Tabela 1). Assim, a hipótese nula de que todos os parâmetros (coeficientes $\beta_{j} j=1,2,3$ ) sejam estatisticamente iguais a zero pode ser rejeitada ao nível de significância de $5 \%$, ou seja, pelos menos uma variável $X$ é estatisticamente significante para explicar a ocorrência. Não houve multicolinearidade entre as variáveis explicativas (Teste VIF abaixo de 5). Após serem atendidos os requisitos para utilizar a regressão do GLM, a Tabela 2 apresenta os resultados das estimativas.

Observa-se na Tabela 2, que os $p$-valores (nível de significância) dos coeficientes $\beta$ das variáveis PIB (2013) per capita, IGM (2016) e a localização dos municípios nas regiões brasileiras apresentaram no Teste de Hipótese de Wald valores inferiores a 0,05 (nível de significância adotado na pesquisa). Isto indica que são significativas no modelo, ou seja, podem ser realizadas as inferências. As variáveis POP e IFDM (Edição 2015 - ano 2013) como apresentaram valores de significância ( $p$-valor) maior que 0,05 não são significativas no modelo.

A variável Índice de Governança Municipal - IGM (2016), por apresentar um coeficiente com sinal positivo $(\beta=1,324)$ e $p$-valor de 0,000 , atendeu às expectativas iniciais. Pode-se concluir, assim, pela rejeição da hipótese nula $\left(\mathrm{H}_{0}\right)$, ou seja, aceita a hipótese alternativa $\left(\mathrm{H}_{1}\right)$ : existe associação (relação) com a variável dependente IFGF. Municípios com maiores escores de governança municipal possuem uma tendência de apresentarem uma melhor gestão fiscal (IFGF). 
Tabela 2 - Resultados das estimativas por regressão do Modelo Linear Generalizado (GLM)

Estimativas do parâmetro por verossimilhança máxima

\begin{tabular}{|c|c|c|c|c|c|c|c|}
\hline \multirow[t]{2}{*}{ Parâmetro } & \multirow[t]{2}{*}{$\beta$} & \multirow{2}{*}{$\begin{array}{l}\text { Modelo } \\
\text { Padrão }\end{array}$} & \multicolumn{2}{|c|}{$\begin{array}{c}\text { Intervalo de confiança de Wald } \\
95 \%\end{array}$} & \multicolumn{3}{|c|}{ Teste de hipótese } \\
\hline & & & Inferior & Superior & $\begin{array}{l}\text { Qui-quadrado } \\
\text { de Wald }\end{array}$ & df & Sig. \\
\hline $\begin{array}{l}\text { (Ordenada na } \\
\text { origem) }\end{array}$ & -0.060 & 0.1937 & -0439 & 0320 & 0095 & 1 & 7 7580 \\
\hline POP & 0,001 & 0,0009 & $-0,001$ & 0,003 & 0,762 & 1 & 0,3830 \\
\hline PIB & $-0,000001903$ & 0,000000929 & $-0,00000372$ & $-0,000000083$ & 4,198 & 1 & 0,040 * \\
\hline IFDM & 0,320 & 0,2978 & $-0,264$ & 0,904 & 1,155 & 1 & 0,2820 \\
\hline IGM & 1,324 & 0,2713 & 0,792 & 1,856 & 23,818 & 1 & 0,000 * \\
\hline NORTE & $-0,282$ & 0,0983 & $-0,475$ & $-0,089$ & 8,236 & 1 & $0,004^{*}$ \\
\hline NORDESTE & $-0,327$ & 0,0895 & $-0,502$ & $-0,151$ & 13,331 & 1 & $0,000^{*}$ \\
\hline SUDESTE & $-0,392$ & 0,0996 & $-0,587$ & $-0,197$ & 15,475 & 1 & $0,000^{*}$ \\
\hline SUL & $-0,383$ & 0,1029 & $-0,584$ & $-0,181$ & 13,831 & 1 & $0,000^{*}$ \\
\hline CENTRO_OESTE & $-0,289$ & 0,0829 & $-0,452$ & $-0,127$ & 12,169 & 1 & $0,000^{*}$ \\
\hline (Escala) & 0,022 & 0,0034 & 0,016 & 0,030 & & & \\
\hline
\end{tabular}

Legenda: $\beta$ = coeficientes; $d f$ = graus de liberdade; Sig. = nível de significância; ${ }^{*}=$ significância dos coeficientes ao nível de $5 \%$.

Fonte: dados da pesquisa.

A localização dos municípios por regiões brasileiras (Sudeste, Sul, Nordeste, Centro-Oeste e Norte), por apresentarem nos coeficientes $\beta$ sinal negativo, não atendeu às expectativas iniciais da investigação (esperava uma relação positiva), mas foram significativos no modelo ( $p$-valor inferior a 0,05$)$. Pode-se concluir, assim, pela rejeição da hipótese nula $\left(\mathrm{H}_{0}\right)$, ou seja, aceita a hipótese alternativa $\left(\mathrm{H}_{1}\right)$ : existe associação (relação) com a variável dependente IFGF. O comportamento dos municípios da amostra é negativo: quanto maior o número de municípios em cada uma das regiões, menor o IFGF. Contudo, essa relação apresenta menores influências na ordem das regiões: Sudeste $(\beta=-0,392)$; Sul $(\beta=-0,383)$; Nordeste $(\beta=-0,327)$, Centro-Oeste $(\beta=-0,289)$ e Norte $(\beta=-0,282)$, ou seja, aumentos de números de municípios, por exemplo, na região Sudeste poderão ter impacto menor que as demais regiões e sucessivamente.

O PIB (2013) per capita apresentou um sinal negativo na relação com a variável IFGF com um nível de significância de 0,04 ( $p$-valor menor que 0,05 ). Assim, não atendeu às expectativas iniciais, mas concluise pela rejeição da hipótese nula $\left(\mathrm{H}_{0}\right)$, ou seja, aceita a hipótese alternativa $\left(\mathrm{H}_{1}\right)$ : existe associação (relação) com a variável dependente IFGF. O comportamento negativo indica que maiores bens e serviços finais produzidos em determinado município, menor seria os escores da gestão fiscal (IFGF).

Os resultados obtidos desta investigação não apresentam cotejamento com a literatura (MENDES; SOUSA, 2006; CRUZ, 2010; GEMMELL; KNELLER; SANZ, 2016; CFA, 2018; TEJEDO-ROMERO; ARAÚJO, 2018) utilizada na escolha das variáveis, haja vista que foram escolhidas como proxies pela falta de estudos empíricos semelhantes. Desse modo, a investigação apresenta uma contribuição inédita (difere os estudos de COSTA; TAVARES; 2014; AVELINO et al., 2014; MELO; SILVA, 2016). Quanto ao uso da Teoria da Agência, observa-se que mesmo não definindo variáveis específicas para serem testadas, os resultados são relevantes para minimizar a assimetria informacional (foco principal da teoria).

\section{Considerações Finais}

Os índices e indicadores utilizados nas avaliações de resultados de ações governamentais são considerados sintéticos, ou seja, avaliam alguns aspectos relacionados com a gestão pública. Contudo, têm suas utilidades no processo avaliativo como artifícios (proxies) para medir inputs, ouptus e outcomes. $O$ desenvolvimento humano é avaliado nas dimensões longevidade, educação e renda com o Índice de Desenvolvimento Humano Municipal (IDHM).

O nível de desenvolvimento socioeconômico local (municípios) é consolidado em um único número através dos resultados obtidos em três vertentes: emprego e renda, educação e saúde pelo Índice FIRJAN de Desenvolvimento Municipal (IFDM). A gestão fiscal dos municípios é avaliada pelo Índice FIRJAN de Gestão Fiscal (IFGF) com base em cinco indicadores: receita própria, gastos com pessoal, investimentos, liquidez e custo da dívida.

Neste sentido, o objetivo desta investigação é analisar, dentre um conjunto de fatores, quais são os explicativos para a gestão fiscal, tendo como proxy o Índice da Federação das Indústrias do Estado do Rio de Janeiro (FIRJAN) de Gestão Fiscal (IFGF) em municípios brasileiros.

Discutir fatores relacionados com a gestão pública é relevante para diminuir a assimetria informacional para a sociedade (principal) que a desconhecem e os gestores públicos (agentes) que a conhecem. As pesquisas avaliativas de resultados de ações governamentais com fulcro na Teoria da Agência (foco na assimetria informacional entre principal-agente) podem cumprir com essa finalidade. Desse modo, tem mais um caso prático de aplicação dessa teoria no setor público. 
Observa-se que as análises deste estudo contribuem para a sociedade (os cidadãos) tomar ciência de fatores relacionados com gestão fiscal, além de contribuir para a literatura com uma forma diferenciada de análises com métrica quantitativa (modelo regressivo generalizado) e assim motivar mais estudos correlatos com outras amostras. Como limitação desta investigação convém ressalvar a confiabilidade dos dados disponibilizados para consulta. Outra limitação do estudo é a utilização de amostra em que os resultados são restritos e não podem ser generalizados para os demais municípios.

Os achados da pesquisa apresentam um avanço na literatura sobre avaliações de resultados de gestão pública. Não foram encontrados estudos empíricos semelhantes para testar as variáveis. Desse modo, a contribuição é inédita e espera que outros estudos sejam realizados em outras amostras para testar as variáveis desta investigação e que também sejam incluídas outras. Convém ressaltar, que as contribuições dos estudos não devem apenas repetir apenas as variáveis mencionadas na literatura. Para avançar, é necessário que o pesquisador teste variáveis que julgar relevantes.

Finalizando, conclui-se que no modelo regressivo utilizado as variáveis do Produto Interno Bruto PIB (2013) per capita, o Índice de Governança Municipal - IGM (2016) e a localização dos municípios nas regiões brasileiras foram significativas para explicar a gestão fiscal em municípios brasileiros. As variáveis número de habitantes (população) e o Índice FIRJAN de Desenvolvimento Municipal IFDM - (Edição 2015 ano 2013) não foram significativas no modelo.

\section{Referências}

AGRESTI, A. An Introduction to Categorical Data Analysis. Second Edition. Hoboken: John Wiley \& Sons, 2007.

ARROW, K. J. The Economics of Agency. Institute for Mathematical Studies in The Social Sciences, Stanford University, California, octuber 1984.

AVELINO, B. C.; CUNHA, J. V. A.; LIMA, G. A. S. F.; COLAUTO, R. D. Características explicativas do nível de disclosure voluntário de municípios do Estado de Minas Gerais. RACE, Unoesc, v. 13, n. 2, p. 571-638, maio/ago. 2014.

BERNARDO, J. S.; REIS, A. O.; SEDIYAMA, G. A. S. Características explicativas do nível de transparência na administração pública municipal. Rev. Ciênc. Admin., Fortaleza, v. 23, n. 2, p. 277-292, maio./ago. 2017. DOI: 10.5020/2318-0722.23.2.277-292

BRASIL. Tribunal de Contas da União. Portaria SEGECEX n 33, de 23 de dezembro de 2010 - Técnica de indicadores de desempenho para auditorias. Brasília, 11 de janeiro de 2011 - Ano XLIV - N. 3.

CAIDEN; G. E.; CAIDEN, N. J. Enfoques y lineamientos para el seguimiento, la medición y la evaluación del desempeño en programas del sector público. Revista do Serviço Público. Ano 52, n.1, p. 78-102, Jan-Mar, 2001. DOI: 10.21874/rsp.v52i1.301

CAVALCANTE, P. Vale a pena ser um bom prefeito? Comportamento eleitoral e reeleição no Brasil. Opinião Pública, Campinas, v. 21, n. 1, abril, 2015, p. 87-104. DOI: 10.1590/1807-019121187.

CFA - Conselho Federal de Administração - Câmara de Gestão Pública - CGP/CFA. Índice de CFA de Governança Municipal - IGM - CFA. Disponível em: http://cgp.cfa.org.br/igm/lista.php. Acesso em: 10 jan. 2018.

CLP - Centro de Liderança Pública. Índice de Oportunidades da Educação Básica (IOEB). Disponível em: http://www.ioeb.org.br//. Acesso em: 10 jan. 2018.

CORDEIRO, G. M.; DEMÉTRIO, C. G. B. Modelos Lineares Generalizados e Extensões. Piracicaba: ESALQ, USP, 2008.

COSTA, F. L.; CASTANHAR, J. C. Avaliação de programas públicos: desafios conceituais e metodológicos. Revista de Administração Pública, Rio de Janeiro, v. 969, n. 92, p.5-37, 2003.

COSTA, T. B.; TAVARES, M. Estudo da relação entre variáveis socioeconômicas e a gestão dos municípios do Estado de Minas Gerais por meio do índice FIRJAN. CONTABILOMETRIA - Brazilian Journal of Quantitative Methods Applied to Accounting, Monte Carmelo, v. 1, n. 2, p. 1-14, 2ํㅗㅇ./2014.

CRUZ, C. F. Transparência da gestão pública municipal: um estudo empírico a partir dos portais eletrônicos dos maiores municípios brasileiros. Rio de Janeiro, 2010. 139f. Dissertação (Mestrado em 
Ciências Contábeis). Faculdade de Administração e Ciências Contábeis, Universidade Federal do Rio de Janeiro, Rio de Janeiro, 2010.

DAWSON, G. S.; DENFORD, J. S.; WILLIAMS, C. K.; PRESTON, D.; DESOUZA, K. C. An Examination of Effective IT Governance in the Public Sector Using the Legal View of Agency Theory. Journal of Management Information Systems, 2016, v. 33, n. 4, p. 1180-1208. DOI:

10.1080/07421222.2016.1267533

FÁVERO, L. P. Análise de dados: modelos de regressão com Excel $\AA_{\text {, }}$ Stata $\circledast$ e SPSS $\circledast$. Rio de Janeiro: Elsevier, 2015.

FIGUEIREDO FILHO, D. B.; PARANHOS, R.; ROCHA, E. C.; SILVA JR, J. A.; MAIA, R. G. Análise de componentes principais para construção de indicadores sociais. Rev. Bras. Biom., São Paulo, v.31, n.1, p. 61-78, 2013.

FIRJAN - Federação das Indústrias do Estado do Rio de Janeiro. Índice FIRJAN de Desenvolvimento Municipal (IFDM). Disponível em: http://www.firjan.com.br/ifdm/. Acesso em: 10 jan. 2018.

FIRJAN - Federação das Indústrias do Estado do Rio de Janeiro. Índice FIRJAN de Gestão Municipal (IFGF). Disponível em: http://www.firjan.com.br/ifgf/.

Acesso em: 10 jan. 2018.

FOLHA DE SÃO PAULO. Ranking de eficiência dos municípios - Folha de São Paulo. Disponível em: http://www1.folha.uol.com.br/remf/. Acesso em 10 jan. 2018. Fernando Canzian, São Paulo.

GEMMELL, N.; KNELLER, R.; SANZ, I. Does the Composition of Government Expenditure Matter for LongRun GDP Levels? Oxford Bulletin of Economics and Statistics, v. 78, n. 4, p. 522-547, 2016.

GUIMARÃES, J. R. S.; JANNUZZI, P. M. IDH, indicadores sintéticos e suas aplicações em políticas públicas: uma análise crítica. R. B. Estudos Urbanos e Regionais, v. 7, p. 73-90, n. 1 / maio 2005. DOI: 10.22296/2317-1529.2005v7n1p7

IBGE - Instituto Brasileiro de Geografia e Estatística. Cidades e Estados. Disponível em: http://cidades.ibge.gov.br/xtras/home.php. Acesso em: 20 jun 2017.

INEP - Instituto Nacional de Estudos e Pesquisas Educacionais Anísio Teixeira. IDEB. Disponível em: http://portal.inep.gov.br/ideb. Acesso em: 10 jan. 2018

JENSEN, M. C.; MECKLING, W. H. Theory of the Firm: Managerial Behavior, Agency Costs and Ownership Structure. Journal of Financial Economics, Harvard, v. 3, n. 4, p. 305-360, out. 1976. DOI: 10.1016/0304405X(76)90026-X

JULNES, P. L. Performance measurement: An effective tool for government accountability? The Debate Goes On, v. 12, n. 2, p. 219-235, 2006. DOI: 10.1177/1356389006066973

KLERING, L. R.; KRUEL, A. J.; STRANZ, E. Os pequenos municípios do Brasil - uma análise a partir de índices de gestão. Análise - Revista de Administração da PUCRS, Porto Alegre, v. 23, n. 1, p. 31-44, jan./abr. 2012.

LERUTH; L.; PAUL, E. A principal-agent theory approach to public expenditure management systems in developing countries. IMF Working Paper, 06/204, September 2006, p. 1-43.

MALMIR, A.; SHIRVANI A.; RASHIDPOUR, A.; SOLTANI, I. Citizen relationship management and principal/agent theory. International Journal of Managing Value and Supply Chains (IJMVSC), v. 5, n. 3 , p. 83-90, September 2014. DOI: 10.5121/ijmvsc.2014.5307.

MARTINS, G. A.; THEÓPHILO, C. R. Metodologia da investigação científica para ciências sociais aplicadas. 2. ed. São Paulo: Atlas, 2009.

MATIAS-PEREIRA, J. Manual de metodologia da pesquisa Científica. 3. ed. São Paulo: Atlas, 2012.

MENDES, C. C.; SOUSA, M. D. C. S. Demand for locally provided public services within the median voter's 
framework: the case of the Brazilian municipalities. Applied Economics, v. 38, n. 3, p. 239-251, 2006. DOI: $10.1080 / 00036840500215378$

MCCULLAGH, P.; NELDER, J. A. Generalized Linear Models. 2nd Edition. London: Chapman \& Hall, 1989.

MELO, C. A. V.; SILVA, L. M. F. Boa Gestão e Políticas Públicas Municipais. Revista de Ciências Sociais. Fortaleza, v.47, n. 2, p.163-196, jul./dez., 2016.

MELO, C. A. V.; SOUZA, S. S.; BONFIM, W. L. S. Federalismo e bons governos: uma análise política da gestão fiscal dos municípios. Opinião Pública, Campinas, v. 21, n. 3, p. 673-692, dezembro, 2015. DOI: 10.1590/1807-01912015213673.

MYERS, R. H.; MONTGOMERY, D. C.; VINING, G. G.; ROBINSON, T. J. Generalized Linear Models with Applications in Engineering and the Sciences. Second Edition. Hoboken: John Wiley \& Sons, 2010.

OLSSON, U. Generalized Linear Models: An Applied Approach. Lund: Studentlitteratur, 2002.

PACHECO, R. S. Mensuração de desempenho no setor público: os termos do debate. Cadernos Gestão Pública e Cidadania, v. 14, oㅡ 55 - jul./dezembro de 2009, p. 149-161.

DOI: $10.12660 /$ cgpc.v14n55.44208.

PEREIRA, M. R.; MENDES, K. V.; CERETTA, P. S.; CORONEL, D. A. Indicadores socioeconômicos e a gestão fiscal dos municípios maranhenses. Revista UNIABEU, Belford Roxo, v. 6, número 14, setembrodezembro 2013.

PNUD - Programa das Nações Unidas para o Desenvolvimento. Índice de Desenvolvimento Humano Municipal (IDHM). Disponível em: http://www.br.undp.org/content/brazil/pt/home/idh0/rankings/idhmmunicipios-2010.htm. Acesso em: 10 jan. 2018.

ROCHA, I.; PEREIRA, A. M.; BEZERRA, F. A.; NASCIMENTO, S. Análise da produção científica sobre teoria da agência e assimetria da informação. REGE - Revista de Gestão, v. 19, n. 2, p. 329-341, 2012. DOI: $10.5700 /$ rege 466 .

SCHILLEMANS, T. Calibrating public sector accountability: translating experimental findings to public sector accountability. Public Management Review, v. 8, p.1-20, Dec 2015.

DOI: $10.1080 / 14719037.2015 .1112423$

SECCHI, L. Políticas públicas: conceitos, esquemas de análise, casos práticos. 2. ed. São Paulo: Cengage Learning, 2013.

SHIKIDA, C. D.; FARIA, F. R. F.; ARAÚJO JR., A. F. Plebiscito e Criação de Novos Estados: o caso do Pará. Economic Analysis of Law Review - EALR, v. 5, n. 1, p. 1-21, Jan-Jun, 2014. DOI: 10.18836/21780587/ealr.v5n1p1-21.

SILVA, M. C.; SILVA, R. A.; SILVA, J. D. G. Análise e avaliação de políticas públicas: aspectos conceituais. Boletim Governet de Administração Pública e Gestão Municipal - n. 61 - outubro/2016 - p. 1434-1444 ISSN 2237-8006 - volume único.

SUZART, J. A. S. As Instituições Superiores de Auditoria: um estudo do nível de transparência fiscal dos países. Contabilidade, Gestão e Governança, Brasília, v. 15, n. 3, p. 107-118, set./dez. 2012.

SWAN, T. Generalized estimating equations when the response variable has a Tweedie distribution: An application for multi-site rainfall modelling. Department of Mathematics and Computing the University of Southern Queensland, Toowoomba, QLD, July 7, 2006.

TEJEDO-ROMERO, F.; ARAÚJO, J. F. F. E. Transparencia en los municipios españoles: determinantes de la divulgación de información". Convergencia Revista de Ciencias Sociales, n. 78, septiembre/diciembre, pp. 153-174, 2018. Universidad Autónoma del Estado de México. DOI: 10.29101/crcs.v25i78.9254

TSHUKUDU, T. T. An integrated performance management model to be used by the Botswana public service sector. Journal of Public Administration and Governance, ISSN 2161-7104, v. 4, n. 2, p. 26-39, 2014. 
TURKMAN, M. A, A.; SILVA, G. L. Modelos Lineares Generalizados: da teoria à prática. Editions SPE, Lisboa, 2000.

\section{NOTAS}

\section{AGRADECIMENTOS}

Não se aplica.

\section{CONTRIBUIÇÃO DE AUTORIA}

Concepção e elaboração do manuscrito: M. C. Silva, F. J. V. Souza, J. D. M. Martins, R. P. B. Câmara

Coleta de dados: M. C. Silva

Análise de dados: M. C. Silva

Discussão dos resultados: M. C. Silva

Revisão e aprovação: M. C. Silva

\section{CONJUNTO DE DADOS DE PESQUISA}

Todo o conjunto de dados que dá suporte aos resultados deste estudo foi publicado no próprio artigo.

\section{FINANCIAMENTO}

Não se aplica.

\section{CONSENTIMENTO DE USO DE IMAGEM}

Não se aplica.

\section{APROVAÇÃO DE COMITÊ DE ÉTICA EM PESQUISA \\ Não se aplica.}

\section{CONFLITO DE INTERESSES}

Não se aplica.

\section{LICENÇA DE USO}

Os Direitos Autorais para artigos publicados neste periódico são do autor, com direitos de primeira publicação para a Revista. Em virtude de aparecerem nesta Revista de acesso público, os artigos são de uso gratuito, com atribuições próprias, em aplicações educacionais, de exercício profissional e para gestão pública. A Revista adotou a licença Creative Commons Atribuição 4.0 Internacional - CC BY NC ND. Esta licença permite acessar, baixar (download), copiar, imprimir, compartilhar, reutilizar e distribuir os artigos desde que com a citação da fonte, atribuindo os devidos créditos de autoria. Nesses casos, nenhuma permissão é necessária por parte dos autores ou dos editores. Autores têm autorização para assumir contratos adicionais separadamente, para distribuição não-exclusiva da versão do trabalho publicada nesta revista (ex.: publicar em repositório institucional ou um capítulo de livro).

\section{PUBLISHER}

Universidade Federal de Santa Catarina. Curso de Ciências Contábeis e Programa de Pós-graduação em Contabilidade. Publicação no Portal de Periódicos UFSC. As ideias expressadas neste artigo são de responsabilidade de seus autores, não representando, necessariamente, a opinião dos editores ou da universidade.

\section{EDITORES}

Carlos Eduardo Facin Lavarda e Suliani Rover

\section{HISTÓRICO}

Recebido em: 30/03/2018 - Revisado por pares em: 22/04/2020 - Reformulado em: 26/04/2020 Recomendado para publicação em: 02/05/2020 - Publicado em: 30/05/2020 